\title{
SPATIAL DECAY ESTIMATES FOR PLANE FLOW IN BRINKMAN-FORCHHEIMER MODEL
}

\author{
BY \\ Y. QIN (Dept. of Mathematics and Statistics, McMaster Univ., Hamilton, Ontario, Canada) \\ AND \\ P. N. KALONI (Department of Mathematics and Statistics, University of Windsor, Windsor, \\ Ontario, Canada)
}

1. Introduction. In comparison to the large amount of work that has been carried out on the analysis of Saint-Venant's principle for solutions of various elliptic boundary problems in elasticity (see $[6,8]$, and the references therein), the related studies to investigate the spatial decay of solutions for flow problems in fluid dynamics are still considerably fewer in the literature. Horgan and Wheeler [11] presented Saint-Venant type results within the general framework of the Navier-Stokes equations governing the steady laminar flow of an incompressible viscous fluid in a cylindrical pipe of arbitrary cross section. These authors studied the classical entry flow problem of viscous laminar flow theory and showed that the decay rate, which depended upon the Reynolds number, the prescribed entry profile of the base flow, and the cross section of the pipe, was exponential. A two-dimensional version of this problem with more explicit results was also investigated by Horgan [9]. Ames and Payne [2] readdressed the problem studied in [11] with a somewhat different approach in formulation of the problem and methodology. They relaxed the assumption that the flow is fully developed at the exit section and obtained more explicit estimates for a weighted energy integral associated with the flow.

It is well known that the stream function in two-dimensional Stokes flow satisfies a biharmonic equation; hence the study of the spatial evolution of stationary Stokes flows in a semi-infinite parallel plate channel has direct relevance to the results of Saint-Venant's principle in plane elastostatics, where the latter problems have been well studied by many authors $[7,13,15,17,19]$. On the other hand, it was shown by a number of investigators $[3,4,10,14]$ that for some initial boundary value problems, the solutions of parabolic equations enjoy the spatial decay behavior of their counterparts for related elliptic equations. A natural question then arises as to whether or not the same exponential spatial decay behavior is present for the solution of transient Stokes flow. The investigations of this question have only been carried out recently. Lin [16] presented a study dealing with

Received February 1, 1995.

1991 Mathematics Subject Classification. Primary 35B45, 35Q30, 76D06.

(C)1998 Brown University 
two-dimensional flow of the time-dependent Stokes equation. The author established an exponential decay estimate for an energy expression associated with the velocity field. The three-dimensional version of the unsteady pipe flow problem was studied recently by Ames, Payne, and Schaefer [1]. These authors developed different techniques from those of [16] for establishing decay results.

In this paper, we investigate the unsteady plane flow through a porous medium in a semi-infinite rectangular strip. We model the porous medium by the BrinkmanForchheimer flow model [5], which is useful in the study of a high porosity porous medium. It is now generally accepted that this model is superior to Darcy's law in that it accounts for the necessary boundary conditions at an interface and the fluid inertia. When the Reynolds number of the flow is greater than unity, it is now suggested that Forchheimer inertia corrections are, generally, needed in the Brinkman equation. As was indicated in [20], in which the spatial decay results in the three-dimensional case were established, the nonlinearity appearing in this model makes the problems considerably more complicated than the Stokes flow problem, although the nonlinear term is of only the quadratic form. Since the plane flow equation has entirely different features, we here present the analogous exponential decay results for the plane Brinkman-Forchheimer flow in a semi-infinite strip.

The plan of the paper is as follows:

In the next section, we formulate the initial boundary problem that serves as the basis for our analysis. After recording, in $\S 3$, some auxiliary results that we use later on, we establish an inequality for the energy expression in $\S 4$. Comparison of the BrinkmanForchheimer flow to the Brinkman flow whose initial boundary data coincide with that of the former is made in $\S 5$. Section 6 is then devoted to a description of how bounds for the total energy in terms of data, the physical parameters, and geometry can be derived.

2. Statement of the problem. Let $R$ be the semi-infinite strip for which $0<x_{1}<$ $\infty, 0<x_{2}<h$. The subdomain of $R$ for which $x_{1}>z$ is denoted by $R_{z}$, and so $R_{0} \equiv R$. The line segment containing the points $\left(z, x_{2}\right)$ is denoted by $L_{z}$ for each $z \geq 0$.

The velocity field $u_{\alpha}\left(x_{1}, x_{2}, t\right)(\alpha=1,2)$ and the pressure $p\left(x_{1}, x_{2}, t\right)$ of the fluid are assumed to be classical solutions of the following initial-boundary problem:

$$
\begin{gathered}
u_{\alpha, t}=\Delta u_{\alpha}-J_{1} u_{\alpha}-J_{2}\left(u_{\beta} u_{\beta}\right)^{1 / 2} u_{\alpha}-p_{, \alpha} \quad \text { in } R \times(0, T), \\
u_{\alpha, \alpha}=0 \quad \text { in } R \times(0, T), \\
u_{\alpha}\left(x_{1}, 0, t\right)=u_{\alpha}\left(x_{1}, h, t\right)=0, \quad \alpha=1,2, \\
u_{\alpha}\left(0, x_{2}, t\right)=g_{\alpha}\left(x_{2}, t\right), \quad \alpha=1,2, \\
u_{\alpha}\left(x_{1}, x_{2}, 0\right)=0, \quad\left(x_{1}, x_{2}\right) \in R,
\end{gathered}
$$

where Eq. (2.1) is the nondimensional form of the Brinkman-Forchheimer equation [5] which describes the flow through a porous medium with consideration of boundary layer and inertial effects, $\Delta$ denotes the Laplace operator, the comma in the subscript denotes partial differentiation, and repeated index convention is used. Here $J_{1}$ and $J_{2}$ are two 
physical parameters (constants), $J_{1}$ being connected with Darcy velocity and $J_{2}$ with the Forchheimer inertial drag term. As was pointed out earlier when the fluid inertia cannot be neglected, it is necessary to augment the Forchheimer drag term to the Brinkman equation. The functions $g_{\alpha}\left(x_{2}, t\right)(\alpha=1,2)$ are assumed to satisfy the compatibility condition $g_{\alpha}(0, t)=g_{\alpha}(h, t)=0$. We remark that in the special case when $J_{1}=0$, $J_{2}=0$, problem (2.1)-(2.5) reduces the Stokes flow equation considered in [16]. For the two-dimensional problems, it is convenient to introduce a stream function $\phi\left(x_{1}, x_{2}, t\right)$ such that

$$
u_{1}=\phi_{, 2}, \quad u_{2}=-\phi_{, 1} .
$$

Using the stream function $\phi\left(x_{1}, x_{2}, t\right)$ and eliminating the pressure in $(2.1)$, we transform problem (2.1)-(2.5) into the initial boundary value problem for a fourth-order equation:

$$
\begin{gathered}
\phi_{, \alpha \alpha t}=\phi_{, \alpha \alpha \beta \beta}-J_{1} \phi_{, \alpha \alpha}-J_{2}\left(\left(\phi_{, \beta} \phi_{, \beta}\right)^{1 / 2} \phi_{, \alpha}\right)_{, \alpha} \text { in } R \times(0, \infty), \\
\phi=\phi_{, 2}=0 \quad \text { for } x_{2}=0, h, t \geq 0, \\
\phi_{, \alpha}\left(0, x_{2}, t\right)=\tilde{g}_{\alpha}\left(x_{2}, t\right), \quad \alpha=1,2, \\
\phi_{, \alpha}\left(x_{1}, x_{2}, 0\right)=0, \quad\left(x_{1}, x_{2}\right) \in R,
\end{gathered}
$$

where $\tilde{g}_{2}=g_{1}, \tilde{g}_{1}=-g_{2}$.

We assume further the following asymptotic behavior for $\phi$ and its derivatives:

$$
\left.\phi, \phi_{, \alpha}, \phi_{, \alpha \beta}, \phi_{, \alpha t} \rightarrow 0 \quad \text { (uniformly in } x_{2}, t\right) \text { as } x_{1} \rightarrow \infty \text {. }
$$

We define an energy integral for the solution $\phi$ of $(2.7)-(2.11)$ by

$$
\begin{aligned}
E(z, t) & =\int_{0}^{t} \int_{R_{z}} \phi_{, \alpha \beta} \phi_{, \alpha \beta} d A d \tau+\frac{h^{2}}{\pi^{2}} \int_{0}^{t} \int_{R_{z}} \phi_{, \alpha \tau} \phi_{, \alpha \tau} d A d \tau \\
& =\int_{0}^{t} \int_{R_{z}}|\nabla u|^{2} d A d \tau+\frac{h^{2}}{\pi^{2}} \int_{0}^{t} \int_{R_{z}}\left|u_{, \tau}\right|^{2} d A d \tau .
\end{aligned}
$$

Physically, the first term on the right-hand side of (2.12) (see [9]) represents a measure of energy dissipation. Following [9], [16] our aim is to establish a differential inequality and then to deduce an exponential decay estimate for the energy expression $E(z, t)$.

3. Auxiliary inequalities. In order to derive a differential inequality for the energy function $E(z, t)$ we shall make frequent use of the Schwarz inequality and the arithmeticgeometric mean $(\mathrm{AG})$ inequality. In addition, we also need the following four inequalities.

(i) If $\phi\left(x_{2}\right) \in C^{1}(0, h)$ and $\phi(0)=\phi(h)=0$, then

$$
\int_{0}^{h} \phi^{2} d x_{2} \leq \frac{h^{2}}{\pi^{2}} \int_{0}^{h} \phi_{, 2}^{2} d x_{2} .
$$

(ii) If $\phi\left(x_{2}\right) \in C^{2}(0, h)$ and $\phi(0)=\phi_{, 2}(0)=0, \phi(h)=\phi_{, 2}(h)=0$, then

$$
\int_{0}^{h} \phi_{, 2}^{2} d x_{2} \leq \frac{h^{2}}{4 \pi^{2}} \int_{0}^{h} \phi_{, 22}^{2} d x_{2} \text {. }
$$


(iii) If $\phi\left(x_{2}\right)$ satisfies the same condition as in ii), then

$$
\int_{0}^{h} \phi^{2} d x_{2} \leq \frac{h^{4}}{\mu_{0}^{4}} \int_{0}^{h} \phi_{, 22}^{2} d x_{2}
$$

where $\mu_{0}$ is a positive constant, a value that is slightly greater than $\frac{3 \pi}{2}$. For simplicity, we may use the inequality below instead of (3.3):

$$
\int_{0}^{h} \phi^{2} d x_{2} \leq\left(\frac{2}{3}\right)^{4} \frac{h^{4}}{\pi^{4}} \int_{0}^{h} \phi_{, 22}^{2} d x_{2}
$$

(iv) If $\phi\left(x_{1}, x_{2}\right) \in C^{1}\left(R^{2}\right)(R=(-\infty, \infty))$ with compact support in $R^{2}$, then

$$
\iint_{-\infty}^{\infty} \phi^{4} d A \leq \frac{1}{2}\left(\iint_{-\infty}^{\infty} \phi^{2} d A\right)\left(\iint_{-\infty}^{\infty} \phi_{, \alpha} \phi_{, \alpha} d A\right) .
$$

The first three inequalities are known as the Wirtinger-type inequality [7], whereas the fourth inequality is a Sobolev inequality $[2,9]$.

4. Energy estimates. In this section, we devote ourselves to establishing the exponential decay estimates for $E(z, t)$. It is convenient to define a weighted energy of the form

$$
\begin{aligned}
\Phi(z, t) & =\int_{0}^{t} \int_{R_{z}}(\xi-z) \phi_{, \alpha \beta} \phi_{, \alpha \beta} d A d \tau+\frac{h^{2}}{\pi^{2}} \int_{0}^{t} \int_{R_{z}}(\xi-z) \phi_{, \alpha \tau} \phi_{, \alpha \tau} d A d \tau \\
& =I_{1}+\frac{h^{2}}{\pi^{2}} I_{2}
\end{aligned}
$$

and we note

$$
E(z, t)=-\frac{\partial \Phi}{\partial z}
$$

On integrating by parts, using Eq. (2.7) and the initial-boundary condition (2.8)-(2.11), we have

$$
\begin{aligned}
& I_{1}=\int_{0}^{t} \int_{R_{z}}(\xi-z) \phi_{, \alpha \beta} \phi_{, \alpha \beta} d A d \tau \\
& =\int_{0}^{t} \int_{R_{z}}(\xi-z) \phi \phi_{, \alpha \alpha \beta \beta} d A d \tau-2 \int_{0}^{t} \int_{R_{z}} \phi_{, \alpha} \phi_{, 1 \alpha} d A d \tau \\
& -\int_{0}^{t} \int_{L_{z}} \phi \phi, 11 d x_{2} d \tau \\
& =\int_{0}^{t} \int_{R_{z}}(\xi-z) \phi\left[\phi_{, \alpha \alpha \tau}+J_{1} \phi_{, \alpha \alpha}+J_{2}\left(\left(\phi_{, \beta} \phi_{, \beta}\right)^{1 / 2} \phi_{, \alpha}\right)_{, \alpha}\right] d A d \tau \\
& -\int_{0}^{t} \int_{L_{z}} \phi \phi_{, 11} d x_{2} d \tau+\int_{0}^{t} \int_{L_{z}} \phi_{, \alpha} \phi_{, \alpha} d x_{2} d \tau
\end{aligned}
$$


A further integration by parts on the first integral on the right side of (4.3) results in

$$
\begin{gathered}
\int_{0}^{t} \int_{R_{z}}(\xi-z) \phi \phi_{, \alpha \alpha \tau} d A d \tau \\
=-\frac{1}{2} \int_{R_{z}}(\xi-z) \phi_{, \alpha} \phi_{, \alpha} d A-\int_{0}^{t} \int_{R_{z}} \phi \phi_{, 1 \tau} d A d \tau \\
J_{1} \int_{0}^{t} \int_{R_{z}}(\xi-z) \phi \phi_{, \alpha \alpha} d A d \tau \\
=-J_{1} \int_{0}^{t} \int_{R_{z}}(\xi-z) \phi_{, \alpha} \phi_{, \alpha} d A d \tau+\frac{1}{2} J_{1} \int_{0}^{t} \int_{L_{z}} \phi^{2} d x_{2} d \tau
\end{gathered}
$$

and

$$
\begin{aligned}
J_{2} \int_{0}^{t} \int_{R_{z}}(\xi-z) \phi\left(\left(\phi_{, \beta} \phi_{, \beta}\right)^{1 / 2} \phi_{, \alpha}\right)_{, \alpha} d A d \tau \\
=-J_{2} \int_{0}^{t} \int_{R_{z}}(\xi-z)\left(\phi_{, \alpha} \phi_{, \alpha}\right)^{3 / 2} d A d \tau \\
-J_{2} \int_{0}^{t} \int_{R_{z}}\left(\phi_{, \beta} \phi_{, \beta}\right)^{1 / 2} \phi \phi_{, 1} d A d \tau .
\end{aligned}
$$

Using (4.4)-(4.6) and dropping three integral terms that are negative, we then can write

$$
\begin{aligned}
I_{1}= & -\int_{0}^{t} \int_{R_{z}} \phi \phi_{, 1 \tau} d A d \tau-J_{2} \int_{0}^{t} \int_{R_{z}}\left(\phi_{, \beta} \phi_{, \beta}\right)^{1 / 2} \phi \phi_{, 1} d A d \tau \\
& -\int_{0}^{t} \int_{L_{z}} \phi \phi_{, 11} d x_{2} d \tau+\int_{0}^{t} \int_{L_{z}} \phi_{, \alpha} \phi_{, \alpha} d x_{2} d \tau+\frac{1}{2} J_{1} \int_{0}^{t} \int_{L_{z}} \phi^{2} d x_{2} d \tau .
\end{aligned}
$$

Making use of the inequality (3.4) and the arithmetic-geometric mean inequality, we are led to

$$
\begin{aligned}
-\int_{0}^{t} \int_{R_{z}} \phi \phi_{, 1 \tau} d A d \tau & \leq\left(\frac{2}{3}\right)^{2} \frac{h^{2}}{\pi^{2}}\left(\int_{0}^{t} \int_{R_{z}} \phi_{, 22}^{2} d A d \tau \int_{0}^{t} \int_{R_{z}} \phi_{, 1 \tau}^{2} d A d \tau\right)^{1 / 2} \\
& \leq \frac{2 h}{9 \pi}\left(\int_{0}^{t} \int_{R_{z}} \phi_{, 22}^{2} d x_{2} d \tau+\frac{h^{2}}{\pi^{2}} \int_{0}^{t} \int_{R_{z}} \phi_{, 1 \tau}^{2} d A d \tau\right) .
\end{aligned}
$$

We can estimate the last three integrals in (4.7):

$$
\begin{aligned}
-\int_{0}^{t} \int_{L_{z}} \phi \phi_{, 11} d x_{2} d \tau+\int_{0}^{t} \int_{L_{z}} \phi_{, \alpha} \phi_{, \alpha} d x_{2} d \tau \\
\leq \frac{2}{9} \frac{h^{2}}{\pi^{2}}\left(\varepsilon_{1} \int_{0}^{t} \int_{L_{z}} \phi_{, 11}^{2} d x_{2} d \tau+\frac{1}{\varepsilon_{1}} \int_{0}^{t} \int_{L_{z}} \phi_{, 22}^{2} d x_{2} d \tau\right) \\
\quad+\frac{h^{2}}{\pi^{2}} \int_{0}^{t} \int_{L_{z}} \phi_{, 12}^{2} d x_{2} d \tau+\frac{h^{2}}{4 \pi^{2}} \int_{0}^{t} \int_{L_{z}} \phi_{, 22}^{2} d x_{2} d \tau
\end{aligned}
$$

and

$$
\frac{J_{1}}{2} \int_{0}^{t} \int_{L_{z}} \phi^{2} d x_{2} d \tau \leq \frac{J_{1}}{2}\left(\frac{2}{3}\right)^{4} \frac{h^{4}}{\pi^{4}} \int_{0}^{t} \int_{L_{z}} \phi_{, 22}^{2} d x_{2} d \tau
$$


For the second integral term in (4.7), we use the Schwarz inequality to obtain

$$
\begin{aligned}
& \int_{0}^{t} \int_{R_{z}}\left(\phi_{, \beta} \phi_{, \beta}\right)^{1 / 2} \phi \phi_{, 1} d A d \tau \\
& \quad \leq \int_{0}^{t}\left(\int_{R_{z}} \phi_{, \beta} \phi_{, \beta} d A\right)^{1 / 2}\left(\int_{R_{z}}\left(\phi \phi_{, 1}\right)^{2} d A\right)^{1 / 2} d \tau \\
& \quad \leq \max _{0 \leq \tau \leq t}\left(\int_{R_{z}} \phi_{, \beta} \phi_{, \beta} d A\right)^{1 / 2} \int_{0}^{5}\left(\int_{R_{z}} \phi^{4} d A\right)^{1 / 4}\left(\int_{R_{z}} \phi_{, 1}^{4} d A\right)^{1 / 4} d \tau .
\end{aligned}
$$

On the other hand, employing Sobolev inequality (3.5), it follows that

$$
\begin{aligned}
\int_{R_{z}} \phi^{4} d A & \leq \frac{1}{2}\left(\int_{R_{z}} \phi^{2} d A\right)\left(\int_{R_{z}} \phi_{, \alpha} \phi_{, \alpha} d A\right) \\
& \leq \frac{1}{2}\left(\frac{2}{3}\right)^{4} \frac{h^{4}}{\pi^{4}}\left(\int_{R_{z}} \phi_{, 22}^{2} d A\right)\left(\int_{R_{z}} \phi_{, \alpha} \phi_{, \alpha} d A\right) \\
& \leq \frac{1}{2}\left(\frac{2}{3}\right)^{4} \frac{h^{6}}{\pi^{6}}\left(\int_{R_{z}} \phi_{, 22}^{2} d A\right)\left(\int_{R_{z}} \phi_{, 12}^{2} d A+\frac{1}{4} \int_{R_{z}} \phi_{, 22}^{2} d A\right) \\
& \leq \frac{1}{2}\left(\frac{2}{3}\right)^{4} \frac{h^{6}}{\pi^{6}}\left[\left(\int_{R_{z}} \phi_{,, 12}^{2} d A\right)^{2}+\frac{1}{2}\left(\int_{R_{z}} \phi_{, 22} d A\right)^{2}\right] \\
& \leq \frac{1}{4}\left(\frac{2}{3}\right)^{4} \frac{h^{6}}{\pi^{6}}\left(\int_{R_{z}} \phi_{, \alpha \beta} \phi_{, \alpha \beta} d A\right)^{2}
\end{aligned}
$$

and

$$
\begin{aligned}
\int_{R_{z}} \phi_{, 1}^{4} d A & \leq \frac{1}{2}\left(\int_{R_{z}} \phi_{, 1}^{2} d A\right)\left(\int_{R_{z}} \phi_{, 1 \alpha} \phi_{, 1 \alpha} d A\right) \\
& \leq \frac{1}{2} \frac{h^{2}}{\pi^{2}}\left(\int_{R_{z}} \phi_{, 12}^{2} d A\right)\left(\int_{R_{z}} \phi_{, 1 \alpha} \phi_{, 1 \alpha} d A\right) \\
& \leq \frac{1}{2} \frac{h^{2}}{\pi^{2}}\left(\int_{R_{z}} \phi_{, \alpha \beta} \phi_{, \alpha \beta} d A\right)^{2} .
\end{aligned}
$$

We remark here that the Sobolev inequality is applicable in the domain $R_{z}$ provided $\phi$ and $\phi_{, 1}$ have already been evenly extended below $x_{1}=z$. In fact, let $\tilde{\phi}$ be an even extension of $\phi$ about $x_{1}=z$, defined by

$$
\tilde{\phi}\left(x_{1}, x_{2}, t\right)= \begin{cases}\phi\left(x_{1}, x_{2}, t\right) & \text { if } x_{1}>z \\ \phi\left(2 z-x_{1}, x_{2}, t\right) & \text { if } x_{1}<z .\end{cases}
$$

Then applying Sobolev inequality (3.5) for the function $\tilde{\phi}\left(x_{1}, x_{2}, t\right)$ yields

$$
\begin{aligned}
& \int_{0}^{h} \int_{-\infty}^{\infty} \tilde{\phi}^{4}\left(x_{1}, x_{2}, t\right) d x_{1} d x_{2} \\
& \quad \leq \frac{1}{2}\left(\int_{0}^{h} \int_{-\infty}^{\infty} \tilde{\phi}^{2}\left(x_{1}, x_{2}, t\right) d x_{1} d x_{2}\right)\left(\int_{0}^{h} \int_{-\infty}^{\infty} \tilde{\phi}_{, \alpha} \tilde{\phi}_{, \alpha} d x_{1} d x_{2}\right) .
\end{aligned}
$$


Noting that

$$
\begin{aligned}
\int_{-\infty}^{\infty} \tilde{\phi}^{4}\left(x_{1}, x_{2}, t\right) d x_{1} & =\int_{z}^{\infty} \phi^{4}\left(x_{1}, x_{2}, t\right) d x_{1}+\int_{-\infty}^{z} \phi^{4}\left(2 z-x_{1}, x_{2}, t\right) d x_{1} \\
& =2 \int_{z}^{\infty} \phi^{4}\left(x_{1}, x_{2}, t\right) d x_{1}
\end{aligned}
$$

and

$$
\begin{aligned}
\int_{-\infty}^{\infty} \tilde{\phi}^{2}\left(x_{1}, x_{2}, t\right) d x_{1} & =2 \int_{z}^{\infty} \phi^{2}\left(x_{1}, x_{2}, t\right) d x_{1} \\
\int_{-\infty}^{\infty} \tilde{\phi}_{, \alpha} \tilde{\phi}_{, \alpha} d x_{1} & =2 \int_{z}^{\infty} \phi_{, \alpha} \phi_{, \alpha} d x_{1}
\end{aligned}
$$

we thus justify that

$$
\int_{R_{z}} \phi^{4} d A \leq \frac{1}{2}\left(\int_{R_{z}} \phi^{2} d A\right)\left(\int_{R_{z}} \phi_{, \alpha} \phi_{, \alpha} d A\right) .
$$

From (4.11)-(4.13), we thus have

$$
\begin{aligned}
& \int_{0}^{t} \int_{R_{z}}\left(\phi_{, \beta} \phi_{, \beta}\right)^{1 / 2} \phi \phi_{, 1} d A d \tau \\
& \quad \leq \frac{2^{1 / 4}}{3} \frac{h}{\pi} \max _{0 \leq \tau \leq t}\left(\int_{R_{z}} \phi_{, \beta} \phi_{, \beta} d A\right)^{1 / 2} \int_{0}^{t} \int_{R_{z}} \phi_{, \alpha \beta} \phi_{, \alpha \beta} d A d \tau .
\end{aligned}
$$

We now turn to estimate $I_{2}$. It is observed that

$$
\begin{aligned}
I_{2}= & \int_{0}^{t} \int_{R_{z}}(\xi-z) \phi_{, \alpha \tau} \phi_{, \alpha \tau} d A d \tau \\
= & -\int_{0}^{t} \int_{R_{z}}(\xi-z) \phi_{, \tau}\left[\phi_{, \alpha \alpha \beta \beta}-J_{1} \phi_{, \alpha \alpha}-J_{2}\left(\left(\phi_{, \beta} \phi_{, \beta}\right)^{1 / 2} \phi_{, \alpha}\right)_{, \alpha}\right] d A d \tau \\
& -\int_{0}^{t} \int_{R_{z}} \phi_{, \tau} \phi_{, 1 \tau} d A d \tau .
\end{aligned}
$$

Integration by parts results in

$$
\begin{gathered}
\int_{0}^{t} \int_{R_{z}}(\xi-z) \phi_{, \tau} \phi_{, \alpha \alpha \beta \beta} d A d \tau \\
=\frac{1}{2} \int_{R_{z}}(\xi-z) \phi_{, \alpha \beta} \phi_{, \alpha \beta} d A \\
\quad+\int_{0}^{t} \int_{R_{z}}\left(2 \phi_{, 1 \tau} \phi_{, 11}+2 \phi_{, 12} \phi_{, 2 \tau}\right) d A d \tau \\
\quad+\int_{0}^{t} \int_{L_{z}} \phi_{, \tau} \phi_{, 11} d x_{2} d \tau, \\
J_{1}^{t} \int_{R_{z}}(\xi-z) \phi_{, \tau} \phi_{, \alpha \alpha} d A d \tau \\
=-\frac{J_{1}}{2} \int_{R_{z}}(\xi-z) \phi_{, \alpha} \phi_{, \alpha}(t) d A-J_{1} \int_{0}^{t} \int_{R_{z}} \phi_{, \tau} \phi_{, 1} d A d \tau
\end{gathered}
$$


and

$$
\begin{aligned}
J_{2} \int_{0}^{t} & \int_{R_{z}}(\xi-z) \phi_{, \tau}\left(\left(\phi_{, \beta} \phi_{, \beta}\right)^{1 / 2} \phi_{, \alpha}\right)_{, \alpha} d A d \tau \\
& =-\frac{J_{2}}{3} \int_{R_{z}}(\xi-z)\left(\phi_{, \beta} \phi_{, \beta}\right)^{3 / 2} d A-J_{2} \int_{0}^{t} \int_{R_{z}}\left(\phi_{, \beta} \phi_{, \beta}\right)^{1 / 2} \phi_{, \tau} \phi_{, 1} d A d \tau .
\end{aligned}
$$

Using (4.16)-(4.18) and dropping three integral terms that are negative, we obtain

$$
\begin{aligned}
I_{2} \leq & -2 \int_{0}^{t} \int_{R_{z}} \phi_{, 1 \alpha} \phi_{, \alpha \tau} d A d \tau-J_{1} \int_{0}^{t} \int_{R_{z}} \phi_{, \tau} \phi_{, 1} d A d \tau \\
& -J_{2} \int_{0}^{t} \int_{R_{z}}\left(\phi_{, \beta} \phi_{, \beta}\right)^{1 / 2} \phi_{, \tau} \phi_{, 1} d A d \tau \\
& -\int_{0}^{t} \int_{L_{z}} \phi_{, \tau} \phi_{, 11} d x_{2} d \tau-\frac{1}{2} \int_{0}^{t} \int_{L_{z}} \phi_{, \tau}^{2} d x_{2} d \tau .
\end{aligned}
$$

In the same manner as we derived (4.8)-(4.14), we can have the following estimates for the integrals over $R_{z}$ :

$$
\begin{aligned}
& -2 \int_{0}^{t} \int_{R_{z}} \phi_{, 1 \alpha} \phi_{, \alpha \tau} d A d \tau \\
& \leq \frac{\pi}{h}\left(\int_{0}^{t} \int_{R_{z}} \phi_{, 1 \alpha} \phi_{, 1 \alpha} d A d \tau+\frac{h^{2}}{\pi^{2}} \int_{0}^{t} \int_{R_{z}} \phi_{, \alpha \tau} \phi_{, \alpha \tau} d A d \tau\right) \\
& J_{1} \int_{0}^{t} \int_{R_{z}} \phi_{, \tau} \phi_{, 1} d A d \tau \\
& \quad \leq \frac{J_{1} h^{2}}{\pi^{2}}\left(\int_{0}^{t} \int_{R_{z}} \phi_{, 2 \tau}^{2} d A d \tau\right)^{1 / 2}\left(\int_{0}^{t} \int_{R_{z}} \phi_{, 12}^{2} d A d \tau\right)^{1 / 2} \\
& \quad \leq \frac{J_{1} h}{2 \pi}\left[\int_{0}^{t} \int_{R_{z}} \phi_{, 12}^{2} d A d \tau+\frac{h^{2}}{\pi^{2}} \int_{0}^{t} \int_{R_{z}} \phi_{, 2 \tau}^{2} d A d \tau\right]
\end{aligned}
$$

and

$$
\begin{aligned}
& -J_{2} \int_{0}^{t} \int_{R_{z}}\left(\phi_{, \beta} \phi_{, \beta}\right)^{1 / 2} \phi_{, \tau} \phi_{, 1} d A d \tau \\
& \quad \leq J_{2} \max _{0 \leq \tau \leq t}\left(\int_{R_{z}} \phi_{, \beta} \phi_{, \beta} d A\right)^{1 / 2} \int_{0}^{t}\left(\int_{R_{z}} \phi_{, 1}^{4} d A\right)^{1 / 4}\left(\int_{R_{z}} \phi_{, \tau}^{4} d A\right)^{1 / 4} d \tau \\
& \quad \leq \frac{J_{2} h}{\sqrt{2} \pi} \max _{0 \leq \tau \leq t}\left(\int_{R_{z}} \phi_{, \beta} \phi_{, \beta} d A\right)^{1 / 2} \int_{0}^{t}\left(\int_{R_{z}} \phi_{, 1 \alpha} \phi_{, 1 \alpha} d A\right)^{1 / 2}\left(\int_{R_{z}} \phi_{, \alpha \tau} \phi_{, \alpha \tau} d A\right)^{1 / 2} d \tau \\
& \quad \leq \frac{J_{2}}{2 \sqrt{2}} \max _{0 \leq \tau \leq t}\left(\int_{R_{z}} \phi_{, \beta} \phi_{, \beta} d A\right)^{1 / 2}\left(\int_{0}^{t} \int_{R_{z}} \phi_{, 1 \alpha} \phi_{, 1 \alpha} d A d \tau\right. \\
& \left.+\frac{h^{2}}{\pi^{2}} \int_{0}^{t} \int_{R_{z}} \phi_{, \alpha \tau} \phi_{, \alpha \tau} d A d \tau\right) .
\end{aligned}
$$


We can also have the estimates for the integrals on the segment $L_{z}$ as

$$
\begin{aligned}
& -\int_{0}^{t} \int_{L_{z}} \phi_{, \tau} \phi_{, 11} d x_{2} d \tau \\
& \quad \leq \frac{1}{2}\left(\varepsilon_{2} \int_{0}^{t} \int_{L_{z}} \phi_{, \alpha \alpha}^{2} d x_{2} d \tau+\frac{1}{\varepsilon_{2}} \frac{h^{2}}{\pi^{2}} \int_{0}^{t} \int_{L_{z}} \phi_{, 2 \tau}^{2} d x_{2} d \tau\right)
\end{aligned}
$$

and

$$
-\frac{1}{2} \int_{0}^{t} \int_{L_{z}} \phi_{, \tau} d x_{2} d \tau \leq \frac{1}{2} \frac{h^{2}}{\pi^{2}} \int_{0}^{t} \int_{L_{z}} \phi_{, 2 \tau}^{2} d x_{2} d \tau .
$$

Summarizing all the above results displayed in (4.3)-(4.24) and choosing $\varepsilon_{1}=\frac{1}{2}$, $\varepsilon_{2}=\frac{7}{5}$, we arrive at

$$
\begin{aligned}
\Phi(z, t) & =I_{1}+\frac{h^{2}}{\pi^{2}} I_{2} \\
& \leq K_{1} E(z, t)+K_{2}\left(-\frac{\partial E}{\partial z}(z, t)\right)
\end{aligned}
$$

where

$$
\begin{gathered}
K_{1}=\frac{11}{9} \frac{h}{\pi}+\frac{J_{1}}{2} \frac{h^{3}}{\pi^{3}}+\left(\frac{2^{1 / 4}}{3}+\frac{1}{2 \sqrt{2}} \frac{h}{\pi}\right) \frac{J_{2} h}{\pi} \max _{0 \leq \tau \leq t}\left(\int_{R_{z}} \phi_{, \beta} \phi_{, \beta} d A\right)^{1 / 2}, \\
K_{2}=\frac{6}{7} \frac{h^{2}}{\pi^{2}}+\frac{J_{1}}{2}\left(\frac{2}{3}\right)^{4} \frac{h^{4}}{\pi^{4}} .
\end{gathered}
$$

Following [9], [16], we can integrate (4.25) by introducing

$$
F(z, t)=e^{-K_{1} z / K_{2}}\left\{E(z, t)+\alpha \int_{z}^{\infty} E(\xi, t) d \xi\right\},
$$

where $\alpha$ is an arbitrary constant to be chosen.

It is easy to show that (4.25) may be rewritten as

$$
\frac{\partial F(z, t)}{\partial z}+\alpha F(z, t) \leq 0
$$

provided $\alpha=\alpha_{0}=\frac{1}{2} \frac{K_{1}}{K_{2}}+\frac{1}{2} \sqrt{\frac{K_{1}^{2}}{K_{2}^{2}}+\frac{4}{K_{2}}}$, which is the positive root of the quadratic equation

$$
\alpha^{2}-\frac{K_{1}}{K_{2}} \alpha-\frac{1}{K_{2}}=0 .
$$

The integration of (4.28) results in

$$
F(z, t) \leq F(0, t) e^{-\alpha_{0} z} .
$$

From (4.31) it can be easily deduced (see [9], [16]) that

$$
E(z, t) \leq \widetilde{K} E(0, t) e^{-\omega z}, \quad z \geq 0
$$

where

$$
\omega=\alpha_{0}-\frac{K_{1}}{K_{2}}=-\frac{1}{2} \frac{K_{1}}{K_{2}}+\frac{1}{2} \sqrt{\frac{K_{1}^{2}}{K_{2}^{2}}+\frac{4}{K_{2}}}
$$




$$
\widetilde{K}=\frac{2 \sqrt{K_{1}^{2}+4 K_{2}}}{\sqrt{K_{1}^{2}+4 K_{2}}-K_{1}} .
$$

Equation (4.32) clearly indicates the exponential decay of energy $E(z, t)$ with the distance $z$ from the end.

It is now of interest to relate our results with those of [16]. We note that if we set $J_{1}=0, J_{2}=0$ in (4.26) and (4.27) and identify $K_{1}=M$ and $K_{2}=\frac{1}{4 K}$ in the above equations, then our results exactly reduce to those of [16]. Further explicit decay rate comparison, however, is not possible at this point since the third term on the right-hand side of (4.26) is not yet estimated. We can, however, demonstrate the following physical implication of (4.32) and (4.33).

In view of the fact that

$$
\begin{aligned}
\frac{\partial \omega}{\partial K_{1}} & =-\frac{1}{2} \frac{1}{K_{2}}+\frac{1}{2}\left(\frac{K_{1}^{2}}{K_{2}^{2}}+\frac{4}{K_{2}}\right)^{-1 / 2} \frac{K_{1}}{K_{2}^{2}} \\
& =+\frac{1}{2 K_{2}}\left(\frac{K_{1}^{2}}{K_{2}^{2}}+\frac{4}{K_{2}}\right)^{-1 / 2}\left[-\left(\frac{K_{1}^{2}}{K_{2}^{2}}+\frac{4}{K_{2}}\right)^{1 / 2}+\frac{K_{1}}{K_{2}}\right]<0
\end{aligned}
$$

and

$$
\begin{aligned}
\frac{\partial \omega}{\partial K_{2}} & =\frac{1}{2} \frac{K_{1}}{K_{2}^{2}}-\frac{1}{2}\left(\frac{K_{1}^{2}}{K_{2}^{2}}+\frac{4}{K_{2}}\right)^{-1 / 2}\left(\frac{K_{1}^{2}}{K_{2}^{3}}+\frac{2}{K_{2}^{2}}\right) \\
& =\frac{1}{2} \frac{K_{1}}{K_{2}^{2}}\left(\frac{K_{1}^{2}}{K_{2}^{2}}+\frac{4}{K_{2}}\right)^{-1 / 2}\left[\left(\frac{K_{1}^{2}}{K_{2}^{2}}+\frac{4}{K_{2}}\right)^{1 / 2}-\left(\frac{K_{1}}{K_{2}}+\frac{2}{K_{1}}\right)\right]<0
\end{aligned}
$$

we see that $\omega$ is a decreasing function of $K_{1}$ and $K_{2}$. Using (4.26) and (4.27), we also see that

$$
\frac{\partial \omega}{\partial J_{1}}=\frac{\partial \omega}{\partial K_{1}} \frac{\partial K_{1}}{\partial J_{1}}+\frac{\partial \omega}{\partial K_{2}} \frac{\partial K_{2}}{\partial J_{1}}<0, \quad \frac{\partial \omega}{\partial J_{2}}=\frac{\partial \omega}{\partial K_{1}} \frac{\partial K_{1}}{\partial J_{2}}<0 .
$$

Thus, $\omega$ is a decreasing function of $J_{1}$ and $J_{2}$. This implies that the decay rate for flow in the B-F model is lower than that for the flow in a pure viscous fluid. We can thus conclude that both the porosity of the medium as well as the inertial drag have the effect of reducing the energy decay rate.

5. Bound for $\max _{0 \leq \tau \leq t} \int_{R_{z}} \phi_{, \beta} \phi_{, \beta} d A$. It was noted in the previous section that the constant $K_{1}$ in (4.26) depends upon the quantity $\max _{0 \leq \tau \leq t} \int_{R_{z}} \phi_{, \beta} \phi_{, \beta} d A$. However, it is important for an a priori estimate that the estimate constants do not depend on the solution $\phi$ of the initial-boundary problem (2.7)-(2.11). Our next task, therefore, is to show that the quantity $\max _{0 \leq \tau \leq t} \int_{R_{z}} \phi_{, \beta} \phi_{, \beta} d A$ has an upper bound that only depends upon the known data, i.e., the initial-boundary data, the physical parameters $J_{1}, J_{2}$, and the strip width $h$. Due to the difficulties caused by the nonlinearity of Eq. (2.7), we are not able to accomplish this in a direct way. Motivated by the idea of treating the problem of the steady Navier-Stokes equation [2, 18], we could, however, compare the solution of the initial-boundary value problem of the nonlinear equation to the solution of the initial-boundary value problem of the corresponding linearized equation. In this manner, we will show that some integral terms involving the solution to the nonlinear 
equations can be controlled by those involving the solution to the linearized equation. In the next section, we will then show that the latter integral terms are bounded above only in terms of the initial-boundary data, physical parameters $J_{1}, J_{2}$, and the strip width $h$.

We consider the following initial boundary value problem:

$$
\begin{gathered}
\psi_{, \alpha \alpha t}=\psi_{, \alpha \alpha \beta \beta}-J_{1} \psi_{, \alpha \alpha} \text { in } R \times(0, \infty), \\
\psi=\psi_{, 2}=0 \quad \text { for } x_{2}=0, h, t \geq 0, \\
\psi_{, \alpha}\left(0, x_{2}, t\right)=\phi_{, \alpha}\left(0, x_{2}, t\right), \quad \alpha=1,2, \\
\psi_{\alpha}\left(x_{1}, x_{2}, 0\right)=0, \quad\left(x_{1}, x_{2}\right) \in R, \\
\left.\psi, \psi_{, \alpha}, \psi_{, \alpha \beta}, \psi_{, \alpha t} \rightarrow 0 \quad \text { (uniformly in } x_{2}, t\right) \quad \text { as } x_{1} \rightarrow \infty .
\end{gathered}
$$

The function $\psi\left(x_{1}, x_{2}, t\right)$ can be regarded as the stream function to the solution of the Brinkman equation whose form is the same as that of Eq. (2.1) when $J_{2}=0$.

We define $\theta=\phi-\psi$. Then $\theta$ is a solution of the following initial boundary-value problem:

$$
\begin{gathered}
\theta_{, \alpha \alpha t}=\theta_{, \alpha \alpha \beta \beta}-J_{1} \theta_{, \alpha \alpha}-J_{2}\left(\left(\phi_{, \beta} \phi_{\beta}\right)^{1 / 2} \phi_{, \alpha}\right)_{, \alpha} \quad \text { in } R \times(0, \infty), \\
\theta=\theta_{, 2}=0 \quad \text { for } x_{2}=0, h, t \geq 0 \\
\theta_{, \alpha}\left(0, x_{2}, t\right)=0, \quad \alpha=1,2, \\
\theta_{, \alpha}\left(x_{1}, x_{2}, 0\right)=0, \quad\left(x_{1}, x_{2}\right) \in R, \\
\left.\theta, \theta_{, \alpha}, \theta_{, \alpha \beta}, \theta_{, \alpha t} \rightarrow 0 \quad \text { (uniformly in } x_{2}, t\right) \quad \text { as } x_{1} \rightarrow \infty .
\end{gathered}
$$

It is easy to verify that (5.8) together with (5.7) also implies $\theta\left(0, x_{2}, t\right)=0$. On multiplying Eq. (5.6) by $\theta$, integrating it by parts over $R \times\left(0, t_{1}\right)$, and using the condition (5.7)-(5.10), for any $0 \leq t_{1} \leq t$ we obtain

$$
\begin{array}{r}
\frac{1}{2} \int_{R} \theta_{, \alpha} \theta_{, \alpha}\left(t_{1}\right) d A+\int_{0}^{t_{1}} \int_{R}\left(\theta_{, \alpha \beta} \theta_{, \alpha \beta}+J_{1} \theta_{, \alpha} \theta_{, \alpha}+\left(\phi_{, \beta} \phi_{, \beta}\right)^{3 / 2}\right) d A d \tau \\
=J_{2} \int_{0}^{t_{1}} \int_{R}\left(\phi_{, \beta} \phi_{, \beta}\right)^{1 / 2} \phi_{, \alpha} \psi_{, \alpha} d A d \tau .
\end{array}
$$


Using the Schwarz and the Sobolev inequalities we estimate the term on the right side of (5.11):

$$
\begin{aligned}
J_{2} \int_{0}^{t_{1}} \int_{R}\left(\phi_{, \beta} \phi_{, \beta}\right)^{1 / 2} \phi_{, \alpha} \psi_{, \alpha} d A d \tau & \\
\leq & J_{2}\left(\int_{0}^{t} \int_{R}\left(\phi_{, \alpha} \phi_{, \alpha}\right)^{2} d A d \tau\right)^{1 / 2}\left(\int_{0}^{t} \int_{R} \psi_{, \alpha} \psi_{, \alpha} d A d \tau\right)^{1 / 2} \\
\leq & \frac{J_{2}}{2}\left(\max _{0 \leq \tau \leq t_{1}} \int_{R} \phi_{, \alpha} \phi_{, \alpha} d A\right)^{1 / 2}\left(\int_{0}^{t} \int_{R} \phi_{, \alpha \beta} \phi_{, \alpha \beta} d A d \tau\right)^{1 / 2} \\
\leq & \frac{J_{2}}{4}\left(\int_{0 \leq \tau \leq t_{1}}^{t} \int_{R} \psi_{, \alpha} \psi_{, \alpha} d A d \tau\right)^{1 / 2} \\
& \left.\quad+\frac{J_{2}}{4} \int_{0}^{t} \int_{R} \phi_{, \alpha \beta} \phi_{, \alpha \beta} d A\right)\left(\int_{0}^{t} \int_{R} \psi_{, \alpha} \psi_{, \alpha} d A d \tau\right)
\end{aligned}
$$

By use of (5.12) in (5.11), we deduce that

$$
\begin{aligned}
\frac{1}{2} \max _{0 \leq \tau \leq t} & \int_{R} \theta_{, \alpha} \theta_{, \alpha} d A+\int_{0}^{t} \int_{R} \theta_{, \alpha \beta} \theta_{, \alpha \beta} d A d \tau \\
\leq & \frac{J_{2}}{4}\left(\max _{0 \leq \tau \leq t} \int_{R} \phi_{, \alpha} \phi_{, \alpha} d A\right)\left(\int_{0}^{t} \int_{R} \psi_{, \alpha} \phi_{, \alpha} d A\right)\left(\int_{0}^{t} \int_{R} \psi_{, \alpha} \psi_{, \alpha} d A d \tau\right) \\
& +\frac{J_{2}}{4} \int_{0}^{t} \int_{R} \phi_{, \alpha \beta} \phi_{, \alpha \beta} d A d \tau .
\end{aligned}
$$

Making use of an elementary inequality $\frac{1}{2} a^{2}-b^{2} \leq(a-b)^{2}$ for two terms on the left side of $(5.13)$, it can be shown that

$$
\begin{aligned}
\frac{1}{4} \max _{0 \leq \tau \leq t} \int_{R} \phi_{, \alpha} \phi_{, \alpha} d A-\frac{1}{2} \max _{0 \leq \tau \leq t} \int_{R} \psi_{, \alpha} \psi_{, \alpha} d A \\
\quad+\frac{1}{2} \int_{0}^{t} \int_{R} \phi_{, \alpha \beta} \phi_{, \alpha \beta} d A d \tau-\int_{0}^{t} \int_{R} \psi_{, \alpha \beta} \psi_{, \alpha \beta} d A d \tau \\
\leq \frac{1}{2} \max _{0 \leq \tau \leq t} \int_{R} \theta_{, \alpha} \theta_{, \alpha} d A+\int_{0}^{t} \int_{R} \theta_{, \alpha \beta} \theta_{, \alpha \beta} d A d \tau .
\end{aligned}
$$

It follows from (5.13) and (5.14) that

$$
\begin{aligned}
\max _{0 \leq \tau \leq t} \int_{R} \phi_{, \alpha} \phi_{, \alpha} d A\left(1-J_{2} \int_{0}^{t} \int_{R} \psi_{, \alpha} \psi_{, \alpha} d A d \tau\right) \\
+\left(2-J_{2}\right) \int_{0}^{t} \int_{R} \phi_{, \alpha \beta} \phi_{, \alpha \beta} d A d \tau \\
\leq 2 \max _{0 \leq \tau \leq t} \int_{R} \psi_{, \alpha} \psi_{, \alpha} d A+4 \int_{0}^{t} \int_{R} \psi_{, \alpha} \psi_{, \alpha} d A d \tau .
\end{aligned}
$$

This implies that the quantities $\max _{0 \leq \tau \leq t} \int_{R} \phi_{, \alpha} \phi_{, \alpha} d A$ and $\int_{0}^{t} \int_{R} \phi_{, \alpha \beta} \phi_{, \alpha \beta} d A d \tau$ can be bounded by the integrals of the solution to the linearized equation, i.e., integrals 
$\max _{0 \leq \tau \leq t} \int_{R} \psi_{, \alpha} \psi_{, \alpha} d A$ and $\int_{0}^{t} \int_{R} \psi_{, \alpha \beta} \psi_{, \alpha \beta} d A d \tau$ provided

$$
\int_{0}^{t} \int_{R} \psi_{, \alpha} \psi_{, \alpha} d A d \tau<\frac{1}{J_{2}} \quad \text { and } \quad J_{2}<2 .
$$

We note that such restrictions are needed in order to derive the desired decay results for solution of nonlinear equations. We remark that similar situations were encountered in [2], [11] for the study of the Navier-Stokes equation and in [20] for the study of the three-dimensional Brinkman-Forchheimer equation.

Next, on integrating by parts we have

$$
\begin{aligned}
\int_{0}^{t} \int_{R} \theta_{, \alpha \tau} \theta_{, \alpha \tau} d A d \tau=-\int_{0}^{t} \int_{R} \theta_{, \tau} \theta_{, \alpha \alpha \tau} d A \\
\leq-\int_{0}^{t} \int_{R} \theta_{, \tau}\left[\theta_{, \alpha \alpha \beta \beta}-J_{1} \theta_{, \alpha \alpha}-J_{2}\left(\left(\phi_{, \beta} \phi_{, \beta}\right)^{1 / 2} \phi_{, \alpha}\right)_{, \alpha}\right] d A d \tau \\
\leq \int_{0}^{t} \int_{R}\left[-\theta_{, \alpha \beta \tau} \theta_{, \alpha \beta}-J_{1} \theta_{, \alpha \tau} \theta_{, \alpha}-J_{2}\left(\left(\phi_{, \beta} \phi_{, \beta}\right)^{1 / 2} \phi_{, \alpha}\right) \theta_{, \alpha \tau}\right] d A d \tau \\
\leq-\int_{R}\left[\frac{1}{2} \theta_{, \alpha \beta} \theta_{, \alpha \beta}+\frac{J_{1}}{2} \theta_{, \alpha} \theta_{, \alpha}+\frac{J_{2}}{3}\left(\phi_{, \beta} \phi_{, \beta}\right)^{3 / 2}\right] d A \\
\quad+J_{2} \int_{0}^{t} \int_{R}\left(\phi_{, \beta} \phi_{, \beta}\right)^{1 / 2} \phi_{, \alpha} \psi_{, \alpha \tau} d A d \tau .
\end{aligned}
$$

Applying the Schwarz and Sobolev inequalities to the last term on the right side of (5.17), we obtain

$$
\begin{gathered}
J_{2} \int_{0}^{t} \int_{R}\left(\phi_{, \beta} \phi_{, \beta}\right)^{1 / 2} \phi_{, \alpha} \psi_{, \alpha \tau} d A d \tau \\
\leq J_{2}\left(\int_{0}^{t} \int_{R}\left(\phi_{, \alpha} \phi_{, \alpha}\right)^{2} d A d \tau\right)^{1 / 2}\left(\int_{0}^{t} \int_{R} \psi_{, \alpha \tau} \psi_{, \alpha \tau} d A d \tau\right)^{1 / 2} \\
\leq \frac{J_{2}}{2}\left(\max _{0 \leq \tau \leq t_{1}} \int_{R} \phi_{, \alpha} \phi_{, \alpha} d A\right)^{1 / 2}\left(\int_{0}^{t} \int_{R} \phi_{, \alpha \beta} \phi_{, \alpha \beta} d A d \tau\right)^{1 / 2} \\
\times\left(\int_{0}^{t} \int_{R} \psi_{, \alpha \tau} \psi_{, \alpha \tau} d A d \tau\right)^{1 / 2}
\end{gathered}
$$

Combining (5.17) and (5.18), we derive

$$
\begin{aligned}
\int_{0}^{t} \int_{R} \phi_{, \alpha \tau} \phi_{, \alpha \tau} d A d \tau & \\
\leq & J_{2}\left(\max _{0 \leq \tau \leq t} \int_{R} \phi_{, \alpha} \phi_{, \alpha} d A\right)^{1 / 2}\left(\int_{0}^{t} \int_{R} \phi_{, \alpha \beta} \phi_{, \alpha \beta} d A d \tau\right)^{1 / 2} \\
& \times\left(\int_{0}^{t} \int_{R} \psi_{, \alpha \tau} \psi_{, \alpha \tau} d A d \tau\right)^{1 / 2}+2 \int_{0}^{t} \int_{R} \psi_{, \alpha \tau} \psi_{, \alpha \tau} d A d \tau .
\end{aligned}
$$

Equation (5.19) together with (5.15) and (5.16) indicates that the integral

$$
\int_{0}^{t} \int_{R} \phi_{, \alpha \tau} \phi_{, \alpha \tau} d A d \tau
$$


is bounded by the integrals

$$
\max _{0 \leq \tau \leq t_{1}} \int_{R} \psi_{, \alpha} \psi_{, \alpha} d A, \quad \int_{0}^{t} \int_{R} \psi_{, \alpha \beta} \psi_{, \alpha \beta} d A d \tau
$$

and

$$
\int_{0}^{t} \int_{R} \psi, \alpha \tau \psi, \alpha \tau d A d \tau
$$

In the next section, we will show that these integrals are also bounded in terms of the known data and physical parameters.

6. Upper bound for the total energy. We first define the function $\omega\left(x_{1}, x_{2}, t\right)$ as

$$
\omega\left(x_{1}, x_{2}, t\right)=\left[\int_{0}^{x_{2}} \tilde{g}(\xi, t) d \xi+x_{1}\left(\tilde{g}_{1}\left(x_{2}, t\right)+r \int_{0}^{x_{2}} \tilde{g}_{2}(\xi, t) d \xi\right)\right] e^{-r x_{1}},
$$

where $r$ is a positive constant to be determined later.

It can be readily shown from (2.8) and (2.9) that $\omega$ satisfies the boundary conditions stated below:

$$
\begin{gathered}
\omega=\omega_{, 2}=0 \text { for } x_{2}=0, h \\
\omega_{, \alpha}\left(0, x_{2}, t\right)=\tilde{g}_{\alpha}\left(x_{2}, t\right), \quad \alpha=1,2, \\
\left.\omega, \omega_{, \alpha}, \omega_{, \alpha \beta} \rightarrow 0 \quad \text { (uniformly in } x_{2}, t\right) \quad \text { as } x_{1} \rightarrow \infty .
\end{gathered}
$$

Moreover, we have

$$
\omega\left(0, x_{2}, t\right)=\int_{0}^{x_{2}} \tilde{g}_{2}(\xi, t) d \xi=\int_{0}^{x_{2}} \phi_{, 2}(0, \xi, t) d \xi=\phi\left(0, x_{2}, t\right) .
$$

Let $\psi\left(x_{1}, x_{2}, t\right)$ be the solution of the problem (5.1)-(5.5) in the previous section. We denote by

$$
\begin{aligned}
E_{\psi \omega}(z, t) & =\int_{0}^{t} \int_{R_{z}} \psi_{, \alpha \beta} \omega_{, \alpha \beta} d A d \tau+\frac{h^{2}}{\pi^{2}} \int_{0}^{t} \int_{R_{z}} \psi_{, \alpha \tau} \omega_{, \alpha \tau} d A d \tau, \\
E_{\omega}(z, t) & =\int_{0}^{t} \int_{R_{z}} \omega_{, \alpha \beta} \omega_{, \alpha \beta} d A d \tau+\frac{h^{2}}{\pi^{2}} \int_{0}^{t} \int_{R_{z}} \omega_{, \alpha \tau} \omega_{, \alpha \tau} d A d \tau .
\end{aligned}
$$

It is clear that

$$
\begin{aligned}
E_{\psi \omega}(0, t)= & \int_{0}^{t} \int_{R} \psi_{, \alpha \beta} \omega_{, \alpha \beta} d A d \tau+\frac{h^{2}}{\pi^{2}} \int_{0}^{t} \int_{R} \psi_{, \alpha \tau} \omega_{, \alpha \tau} d A d \tau \\
= & E_{\psi}(0, t)+\int_{0}^{t} \int_{R} \psi_{, \alpha \beta}\left(\omega_{, \alpha \beta}-\psi_{, \alpha \beta}\right) d A d \tau \\
& \quad+\frac{h^{2}}{\pi^{2}} \int_{0}^{t} \int_{R_{z}} \psi_{, \alpha \tau}\left(\omega_{, \alpha \tau}-\psi_{, \alpha \tau}\right) d A d \tau \\
= & E_{\psi}(0, t)+I_{1}+I_{2} .
\end{aligned}
$$


Making use of the divergence theorem, Eq. (5.6), and the boundary conditions (5.7)(5.10), together with (6.2)-(6.4), we obtain

$$
\begin{aligned}
I_{1}= & \int_{0}^{t} \int_{R} \psi_{, \alpha \alpha \beta \beta}(\omega-\psi) d A d \tau \\
= & \int_{0}^{t} \int_{R}\left(\psi_{, \alpha \alpha \tau}+J_{1} \psi_{, \alpha \alpha}\right)(\omega-\psi) d A d \tau \\
= & -\int_{0}^{t} \int_{R}\left(\psi_{, \alpha \tau}+J_{1} \psi_{, \alpha}\right)\left(\omega_{, \alpha}-\psi_{, \alpha}\right) d A d \tau \\
= & \int_{0}^{t} \int_{R}\left(\psi_{, \alpha \tau} \psi_{, \alpha}+J_{1} \psi_{, \alpha} \psi_{, \alpha}\right) d A d \tau \\
& \quad-\int_{0}^{t} \int_{R}\left(\psi_{, \alpha \tau} \omega_{, \alpha}+J_{1} \psi_{, \alpha} \omega_{, \alpha}\right) d A d \tau \\
\geq & \frac{1}{2} \int_{R} \psi_{, \alpha} \psi_{, \alpha}(t) d A+J_{1} \int_{0}^{t} \int_{R} \psi_{, \alpha} \psi_{, \alpha} d A d \tau \\
& \quad-\frac{1}{4} \frac{h^{2}}{\pi^{2}} \int_{0}^{t} \int_{R} \psi_{, \alpha \tau} \psi_{, \alpha \tau} d A d \tau-\int_{0}^{t} \int_{R} \omega_{, \alpha 2} \omega_{, \alpha 2} d A d \tau \\
& \quad-\frac{J_{1}}{2} \int_{0}^{t} \int_{R} \psi_{, \alpha} \psi_{, \alpha} d A d \tau-\frac{J_{1}}{2} \int_{0}^{t} \int_{R} \omega_{, \alpha} \omega_{, \alpha} d A d \tau,
\end{aligned}
$$

where the Schwarz and AG inequalities have been used to derive the results in the last step.

Similarly, we have

$$
\begin{aligned}
I_{2}= & -\frac{h^{2}}{\pi^{2}} \int_{0}^{t} \int_{R} \psi_{, \alpha \alpha \tau}\left(\omega, \tau-\psi_{, \tau}\right) d A d \tau \\
= & -\frac{h^{2}}{\pi^{2}} \int_{0}^{t} \int_{R}\left(\psi_{, \alpha \alpha \beta \beta}-J_{1} \psi_{, \alpha \alpha}\right)\left(\omega_{, \tau}-\psi_{, \tau}\right) d A d \tau \\
= & \frac{h^{2}}{\pi^{2}} \int_{0}^{t} \int_{R}\left(\psi_{, \alpha \beta} \psi_{, \alpha \beta \tau}+J_{1} \psi_{, \alpha} \psi_{, \alpha \tau}\right) d A d \tau \\
& \quad-\frac{h^{2}}{\pi^{2}} \int_{0}^{t} \int_{R}\left(\psi_{, \alpha \beta} \omega_{, \alpha \beta \tau}+J_{1} \psi_{, \alpha} \omega_{, \alpha \tau}\right) d A d \tau \\
\geq & \frac{1}{2} \frac{h^{2}}{\pi^{2}} \int_{R} \psi_{, \alpha \beta} \psi_{, \alpha \beta \tau}(t) d A+\frac{J_{1}}{2} \frac{h^{2}}{\pi^{2}} \int_{0}^{t} \int_{R} \psi_{, \alpha} \psi_{, \alpha} d A d \tau \\
& \quad-\frac{1}{4} \int_{0}^{t} \int_{R} \psi_{, \alpha \beta} \psi_{, \alpha \beta} d A d \tau-\frac{h^{4}}{\pi^{4}} \int_{0}^{t} \int_{R} \omega_{, \alpha \beta \tau} \omega_{, \alpha \beta \tau} d A d \tau \\
& \quad-\frac{J_{1}}{4} \int_{0}^{t} \int_{R} \psi_{, \alpha} \psi_{, \alpha} d A d \tau-\frac{h^{4}}{\pi^{4}} J_{1} \int_{0}^{t} \int_{R} \omega_{, \alpha \tau} \omega_{, \alpha \tau} d A d \tau
\end{aligned}
$$


Using the Schwarz inequality and combining (6.8)-(6.10), we are led to

$$
\begin{aligned}
\sqrt{E_{\psi}(0, t) E_{\omega}(0, t)} \geq & E_{\psi \omega}(0, t) \\
\geq & \frac{3}{4} E_{\psi}(0, t)+\frac{J_{1}}{4} \int_{0}^{t} \int_{R} \psi_{, \alpha} \psi_{, \alpha} d A d \tau \\
& +\frac{1}{2}\left(1+\frac{J_{1} h^{2}}{\pi^{2}}\right) \int_{R} \psi_{, \alpha} \psi_{, \alpha}(t) d A \\
& -\frac{J_{1}}{2} \int_{0}^{t} \int_{R} \omega_{, \alpha} \omega_{, \alpha} d A d \tau-\int_{0}^{t} \int_{R} \omega_{, \alpha 2} \omega_{, \alpha 2} d A d \tau \\
& -\frac{h^{4}}{\pi^{4}} J_{1} \int_{0}^{t} \int_{R} \omega_{, \alpha \tau} \omega_{, \alpha \tau} d A d \tau-\frac{h^{4}}{\pi^{4}} \int_{0}^{t} \int_{R} \omega_{, \alpha \beta \tau} \omega_{, \alpha \beta \tau} d A d \tau .
\end{aligned}
$$

We then deduce from (6.11) that

$$
\begin{aligned}
\frac{1}{2}(1+ & \left.\frac{J_{1} h^{2}}{\pi^{2}}\right) \int_{R} \psi_{, \alpha} \psi_{, \alpha}(t) d A+\frac{J_{1}}{4} \int_{0}^{t} \int_{R} \psi_{, \alpha} \psi_{, \alpha} d A d \tau \\
& +\frac{3}{4}\left(\sqrt{E_{\psi}(0, t)}-\frac{2}{3} \sqrt{E_{\omega}(0, t)}\right)^{2} \\
\leq & \frac{1}{3} E_{\omega}(0, t)+Q_{\omega}(0, t)
\end{aligned}
$$

where

$$
\begin{aligned}
Q_{\omega}(0, t)= & \frac{J_{1}}{2} \int_{0}^{t} \int_{R} \omega_{, \alpha} \omega_{, \alpha} d A d \tau+\int_{0}^{t} \int_{R} \omega_{, \alpha 2} \omega_{, \alpha 2} d A d \tau \\
& +\frac{h^{4}}{\pi^{4}} J_{1} \int_{0}^{t} \int_{R} \omega_{, \alpha \tau} \omega_{, \alpha \tau} d A d \tau+\frac{h^{4}}{\pi^{4}} \int_{0}^{t} \int_{R} \omega_{, \alpha \beta \tau} \omega_{, \alpha \beta \tau} d A d \tau .
\end{aligned}
$$

Observing $\left(\sqrt{E_{\psi}(0, t)}-\frac{2}{3} \sqrt{E_{\omega}(0, t)}\right)^{2} \geq \frac{1}{2} E_{\psi}(0, t)-\frac{4}{9} E_{\omega}(0, t)$, we can rewrite $(6.12)$ as

$$
\begin{aligned}
\frac{1}{2}\left(1+\frac{J_{1} h^{2}}{\pi^{2}}\right) \int_{R} \psi_{, \alpha} \psi_{, \alpha}(t) d A+\frac{J_{1}}{4} \int_{0}^{t} \int_{R} \psi_{, \alpha} \psi_{, \alpha} d A d \tau & \\
& +\frac{3}{8} E_{\psi}(0, t) \leq \frac{2}{3} E_{\omega}(0, t)+Q_{\omega}(0, t) .
\end{aligned}
$$

It is obvious that

$$
\frac{1}{2}\left(1+\frac{J_{1} h^{2}}{\pi^{2}}\right) \int_{R} \psi_{, \alpha} \psi_{, \alpha}(t) d A \leq \frac{2}{3} E_{\omega}(0, t)+Q_{\omega}(0, t)
$$

and this inequality still holds if $t$ is replaced by $\tau$ on both sides. Therefore, we conclude, for any $0 \leq \tau \leq t$, that

$$
\frac{1}{2}\left(1+\frac{J_{1} h^{2}}{\pi^{2}}\right) \max _{0 \leq \tau \leq t} \int_{R} \psi_{, \alpha} \psi_{, \alpha}(\tau) d A \leq \frac{2}{3} E_{\omega}(0, t)+Q_{\omega}(0, t) .
$$

By choosing an appropriate value of $r$, for instance $r=1$, the results (6.13) and (6.14) clearly show that the quantities $\max _{0 \leq \tau \leq t} \int_{R} \psi_{, \alpha} \psi_{, \alpha}(\tau) d A, \int_{0}^{t} \int_{R} \psi_{, \alpha} \psi_{, \alpha} d A d \tau$, and $E_{\psi}(0, t)$ are bounded in terms of the initial-boundary data, physical parameters $J_{1}, J_{2}$, and the strip width $h$. These results together with the results of the previous 
section indicate that the total energy expression $E(0, t)$ is bounded in the same sense although the bounded expression is not determined here explicitly.

Acknowledgements. The work of Y. Qin is supported by the Postdoctoral Fellowship of N.S.E.R.C. of Canada. The work of P. N. Kaloni is supported by Grant No. A7728 of N.S.E.R.C. of Canada. The authors gratefully acknowledge the support they have received.

\section{REFERENCES}

[1] K. A. Ames, L. E. Payne, and P. W. Schaefer, Spatial decay estimates in time-dependent Stokes flow, SIAM J. Math. Anal. 24, 1395-1413 (1993)

[2] K. A. Ames and L. E. Payne, Decay estimates in steady pipe flow, SIAM J. Math. Anal. 20, 789-815 (1989)

[3] B. A. Boley, Some observations on Saint-Venant's principle, Proc. 3rd U.S. Nat. Cong. Appl. Mech., Brown University, Providence, RI, June 11-14, 1958, American Society of Mechanical Engineers, New York, 1958, pp. 259-264

[4] B. A. Boley, Upper bounds and Saint-Venant's principle in transient heat conduction, Quart. Appl. Math. 18, 205-207 (1960)

[5] R. C. Gilver and S. A. Altobelli, A determination of effective viscosity for the BrinkmanForchhiemer flow model, J. Fluid Mech. 258, 355-370 (1994)

[6] C. O. Horgan, Recent developments concerning Saint-Venant's principle: An update, Appl. Mech. Rev. 42, 295-303 (1989)

[7] C. O. Horgan, Decay estimates for the biharmonic equation with applications to Saint-Venant principles in plane elasticity and Stokes flow, Quart. Appl. Math. 42, 147-157 (1989)

[8] C. O. Horgan and J. K. Knowles, Recent developments concerning Saint-Venant's principle, Adv. Appl. Mech. 23, 179-269 (1983)

[9] C. O. Horgan, Plane entry flows and energy estimates for the Navier-Stokes equations, Arch. Rational Mech. Anal. 68, 359-381 (1978)

[10] C. O. Horgan, L. E. Payne, and L. T. Wheeler, Spatial decay estimates in transient heat conduction, Quart. Appl. Math. 42, 119-127 (1984)

[11] C. O. Horgan and L. T. Wheeler, Spatial decay estimates for the Navier-Stokes equations with application to the problem of entry flow, SIAM J. Appl. Math. 35, 97-116 (1978)

[12] C. T. Hsu and P. Cheng, Thermal dispersion in a porus medium, Internat. J. Heat Mass Transfer 33, 1587-1597 (1990)

[13] J. K. Knowles, On Saint-Venant's principle in the two dimensional linear theory of elasticity, Arch. Rational Mech. Anal. 21, 1-22 (1966)

[14] J. K. Knowles, On the spatial decay of solutions of the heat equation, J. Appl. Math. Phys. (ZAMP) 22, 1050-1056 (1971)

[15] J. K. Knowles, An energy estimate for the biharmonic equation and its application to Saint-Venant's principle in plane elastostatics, Indian J. Pure Appl. Math. 14, 791-805 (1983)

[16] C. Lin, Spatial decay estimates and energy bounds for the Stokes flow equation, SAACM 2, 249-264 (1992)

[17] O. A. Oleinik and G. A. Yosfian, The Saint-Venant's principle in the two-dimensional theory of elasticity and boundary problems for a biharmonic equation in unbounded domains, Sibirsk. Math. Zh. 19, 1154-1165 (1978); English transl. in Siberian Math. J. 19, 813-822 (1978)

[18] L. E. Payne, Uniqueness criteria for steady state solutions of the Navier-Stokes equations, Simpos. Internaz. Appl. Anal. Fis. Math. (Cagliari-Sassari, 1964) Edizioni Cremonese, Rome, 1965, pp. 130-153

[19] L. E. Payne and P. W. Schaefer, Some Phragmén-Lindelof type results for the biharmonic equation, Z. Angew. Math. Phys. (ZAMP) 45, 415-432 (1994)

[20] Y. Qin and J. Chadam, Spatial decay for flow in a porous medium, preprint 